\title{
Visualization of Aneurysmal Neck and Dome after Coiling with 3D Multifusion Imaging of Silent MRA and FSE-MR Cisternography
}

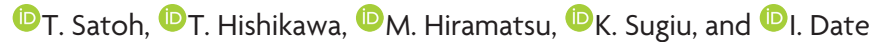

\begin{abstract}
BACKGROUND AND PURPOSE: Our aim was to visualize the precise configuration of the aneurysmal neck and dome with/without remnants combined with a coiled dome after coiling treatment for cerebral aneurysms. We developed 3D multifusion imaging of silent MRA and FSE-MR cisternography.
\end{abstract}

MATERIALS AND METHODS: We examined 12 patients with 3D multifusion imaging by composing 3D images reconstructed from TOF-MRA, silent MRA, and FSE-MR cisternography. The influence of magnetic susceptibility artifacts caused by metal materials affecting the configuration of the aneurysmal complex with coiling was assessed in a single 3D image.

RESULTS: In all cases, TOF-MRA failed to depict the aneurysmal neck complex precisely due to metal artifacts, whereas silent MRA delineated the neck and parent arteries at the coiled regions without serious metal artifacts. FSE-MR cisternography depicted the shape of the coiled aneurysmal dome and parent artery complex together with the brain parenchyma. With the 3D multifusion images of silent MRA and FSE-MR cisternography, the morphologic status of the coiled neck and parent arteries was clearly visualized with the shape of the dome in a single $3 \mathrm{D}$ image.

CONCLUSIONS: Silent MRA is a non-contrast-enhanced form of MRA. It depicts the coiled neck complex without serious metal artifacts. FSE-MR cisternography can delineate the shape of the coiled dome. In this small feasibility study, 3D multifusion imaging of silent MRA and FSE-MR cisternography allowed good visualization of key features of coiled aneurysms. This technique may be useful in the follow-up of coiled aneurysms.

ABBREVIATIONS: AComA = anterior communicating artery; MRC = MR cisternography

l: the follow-up after the endovascular treatment of a cerebral aneurysm, the precise visualization of the shape of the neck and dome with/without remnants from coiling is necessary. ${ }^{1-11} \mathrm{X}$-ray DSA is a reference standard to examine the above-described configuration, but it is invasive and presents certain risks related to the catheter procedure, contrast media,

Received December 8, 2018; accepted after revision March 11, 2019

From the Department of Neurological Surgery (T.S.), Ryofukai Satoh Neurosurgical Hospital, Fukuyama, Hiroshima, Japan; and Department of Neurological Surgery (T.H., M.H., K.S., I.D.), Okayama University Graduate School of Medicine, Okayama, Okayama, Japan.

Paper previously presented, in part, at: Scientific Conference on Neurosurgical Topics of Okayama University Neurosurgical Alumni Association, November 11, 2017; Okayama, Japan; and the Chugoku-Shikoku Scientific Meeting of the Japan Neurosurgical Society, December 8, 2018; Ehime, Japan.

Please address correspondence to Toru Satoh, MD, Department of Neurological Surgery, Ryofukai Satoh Neurosurgical Hospital, 5-23-23 Matsunaga, Fukuyama, Hiroshima 729-0104, Japan; e-mail: ucsfbtrc@urban.ne.jp

-- Indicates open access to non-subscribers at www.ajnr.org

$\equiv$ Indicates article with supplemental on-line table.

http://dx.doi.org/10.3174/ajnr.A6026 and radiation. ${ }^{2,5-7,9,10}$ As a noninvasive substitute for DSA, 3D TOF-MRA with/without contrast media is widely used, but this method presents some difficulty regarding magnetic susceptibility artifacts from the coils. ${ }^{2,4,5,7-11}$

With some recent advances in MR imaging, it has been speculated that silent MRA with zero-TE and arterial spin-labeling tagging may overcome the above problems by delineating flowing blood at the neck with minimal artifacts from metal substances. ${ }^{2,7,9,10,12}$ In addition, MR cisternography (MRC) with a heavily T2-weighted 3D FSE sequence can depict the shape of the aneurysmal dome together with perianeurysmal anatomic structures, including the brain parenchyma and cranial nerves. ${ }^{13-15}$ In the present study, we used 3D multifusion imaging with silent MRAinstead of TOF-MRA — and FSE-MRC to delineate the configuration of the aneurysmal neck and dome after coiling treatment of cerebral aneurysms. To the best of our knowledge, the delineation of the coiled neck with 3D silent MRA and the dome with 3D FSE-MRC, and $3 \mathrm{D}$ multifusion imaging of these structures in a single 3D image has not been described. 


\section{MATERIALS AND METHODS}

This retrospective study was approved by the ethics committee of Ryofukai Satoh Neurosurgical Hospital. Informed consent was obtained from all patients for the use of their clinical data.

\section{Patient Population}

Between July 2017 and June 2018, twelve patients were examined by TOF-MRA, silent MRA, and FSE-MRC for the follow-up of endovascular treatment of a ruptured and/or unruptured cerebral aneurysm at Ryofukai Satoh Neurosurgical Hospital and Okayama University Hospital (On-line Table). The aneurysm location was the anterior communicating artery (AComA) in 5 patients, the distal anterior cerebral artery in 1 patient, the internal carotid-posterior communicating artery in 1 patient, the internal carotid-anterior choroidal artery in 1 patient, the middle cerebral artery in 1 patient, and the top of the basilar artery in 3 patients.

\section{Imaging Procedures}

The imaging by TOF-MRA, silent MRA, and FSE-MRC was performed in the same session on a $3 \mathrm{~T}$ unit (Signa Pioneer; GE Healthcare, Milwaukee, Wisconsin). The TOF-MRA was performed with the following parameters: TR/TE, 25/3.4 ms; flip angle, $20^{\circ}$; FOV , $180 \times 180 \mathrm{~mm}$; matrix, $288 \times 192$; section thickness, $1.2 \mathrm{~mm}$; NEX, 1; bandwidth, $35.71 \mathrm{kHz}$; acquisition time, 5 minutes 41 seconds. A total of 144 continuous axial source images were acquired.

The silent MRA was performed by a silent scan with the following parameters: TR/TE, 880/0.016 ms; flip angle, 5 FOV, $180 \times 180 \mathrm{~mm}$; matrix, $200 \times 200$; spokes per segment, 320; section thickness, $1.0 \mathrm{~mm}$; NEX, 1.5; bandwidth, $31.25 \mathrm{kHz}$; acquisition time, 12 minutes 48 seconds. A total of 400 continuous axial source images were acquired.

The heavily T2-weighted 3D FSE-MRC was performed with the following parameters: TR/TE, 1900/100 ms; FOV, $180 \times 180$ mm; matrix, $356 \times 256$; section thickness, $1.2 \mathrm{~mm}$; NEX, 1; bandwidth, $31.25 \mathrm{kHz}$; echo-train length, 128; acquisition time, $5 \mathrm{~min}$ utes 53 seconds. A total of 60 continuous axial source images were acquired.

\section{Image Processing}

Volumetric datasets from the source images of the TOF-MRA, silent MRA, and FSE-MRC were transferred to a workstation (Ziostation-2; Ziosoft/AMIN, Tokyo, Japan) as described by Satoh et al. ${ }^{13-15}$ For the 3D TOF-MRA and silent MRA, the vessel configurations were reconstructed by a perspective volume-rendering algorithm, and the arterial lumen was selected from the opacity chart of the datasets with higher MR signal intensity of arteries (threshold, $>400$ for TOF-MRA and $>300$ for silent MRA, respectively) compared with the background. Thus, the 3D image of parent arteries and aneurysmal neck after coiling was depicted by the 2 types of MRA.

The perspective volume-rendering image of the 3D FSE-MRC was reconstructed from the entire dataset with a lower signal intensity of CSF (threshold, <1200) so that the shape of the coiled aneurysmal dome and parent artery complex was delineated with its perianeurysmal anatomic structures, including the brain parenchyma and cranial nerves.
To visualize the 2 types of MRA images in combination with MRC images in a single 3D image, we used 3D multifusion imaging by compositing the above-described reconstructed $3 \mathrm{D}$ images of TOF-MRA, silent MRA, and FSE-MRC. The overall time required to reconstruct a picture of $3 \mathrm{D}$ multifusion imaging was within 120 seconds per image. We were thus able to directly compare the shape of the aneurysmal neck complex with/without neck remnants depicted by the 2 types of MRA combined with the coiled dome shown by MRC. The difference between the TOFMRA and silent MRA, in combination with FSE-MRC, was directly compared in each case.

\section{Image-Quality Analysis of TOF-MRA and Silent MRA}

The source images, MIP, 3D perspective volume-rendering, and 3D multifusion images between the TOF-MRA and the silent MRA combined with FSE-MRC were compared with respect to the configuration of the coiled aneurysmal complex depicted by DSA obtained after the completion of the coiling. The 2 types of MRA images were independently reviewed by 2 board-certified neurosurgeons (T.H. and M.H.). The quality of the visualization of the coiled aneurysmal complex including the neck and parent arteries obtained by the TOF-MRA and silent MRA in conjunction with the FSE-MRC was rated subjectively on the following 4-point scale: 1, not visible (no signal or gap in the parent artery or the neck); 2, poor (visible, but incomplete with artifacts); 3 , good (good quality with minimal artifacts); 4, excellent (excellent quality without artifacts). The scores obtained by the 2 observers were averaged for each of the 2 types of MRA, and the statistical analysis was performed by a Wilcoxon signed rank test. A $P$ value $<.05$ was considered significant. Interobserver agreement between the 2 observers' scores for each type of MRA was tested by intraclass correlation coefficient statistics.

\section{Size and Volume Analysis of Coiled Aneurysms}

We measured the maximum size $(\mathrm{x}, \mathrm{y}, \mathrm{z})$ of the aneurysmal neck remnants based on the source images obtained by TOF-MRA and silent MRA and the dome from FSE-MRC in each case. The volume of the remnant was calculated on the basis of ROIs drawn on the source images of TOF-MRA and silent MRA, where the contours of each remnant were respectively serially masked. The voxel volume was determined by a pixel size on the source image and the slice pitch, and the number of voxels within the masked region was counted. The volume of the masked region was then calculated as the number of voxels multiplied by the volume of voxels within the region using a workstation. Similarly, the volume of the dome was calculated on the source images of FSE-MRC. Each numeric value was determined as the mean of 3 measurements performed by the senior author (T.S.).

\section{RESULTS}

\section{Quality Assessment}

The On-line Table provides the data of the 12 patients, including age (62.8 \pm 8.9 years; range, $51-78$ years) and sex ( 4 men and 8 women), location of aneurysms, symptoms, time interval between DSA and MRA (8.5 \pm 4.4 months; range, 3-12 months), and the scores for TOF-MRA and silent MRA (TOF/silent MRAs) obtained by the 2 observers. In all cases, the Wilcoxon signed rank 


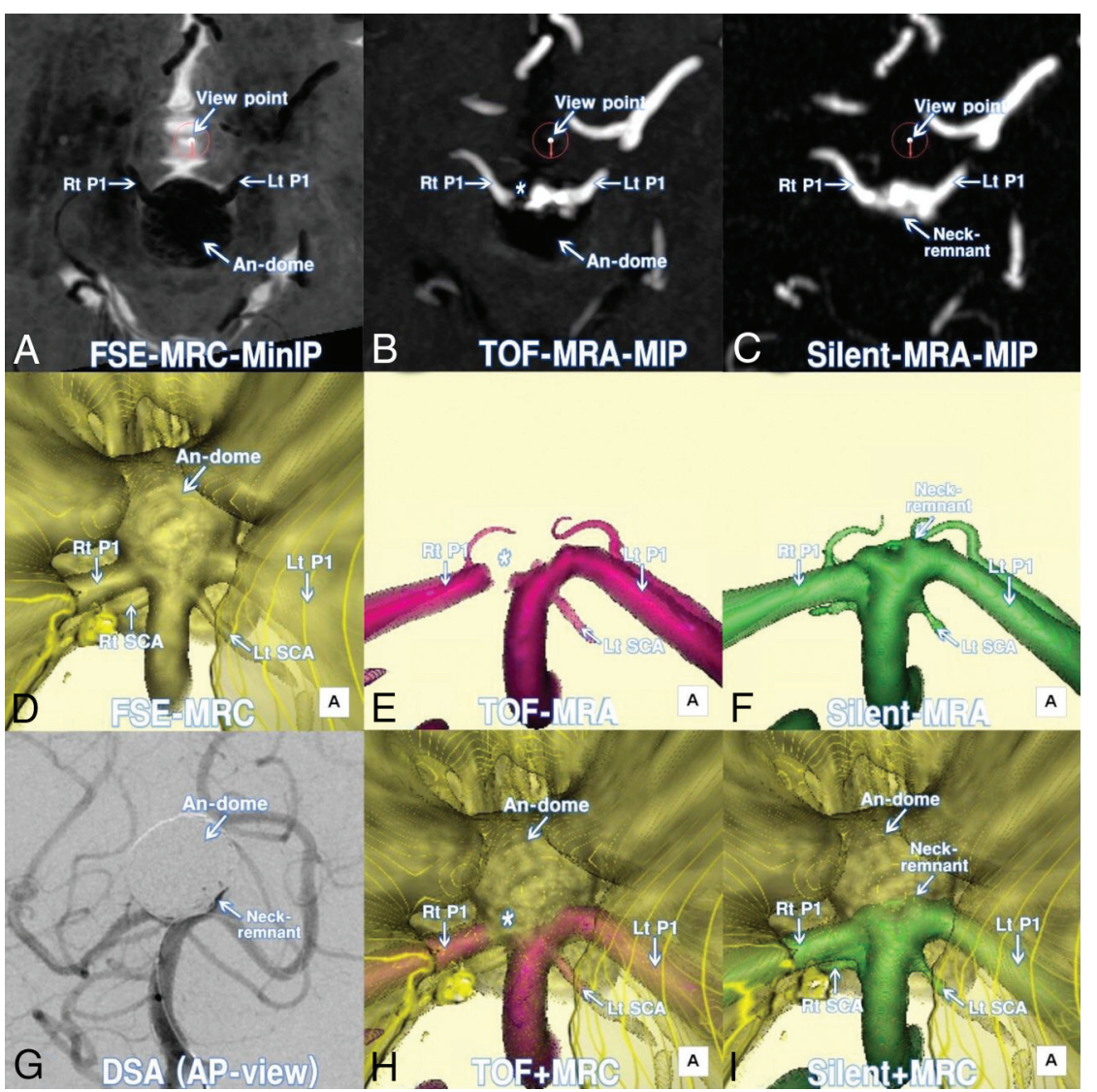

FIG 1. A 69-year-old woman (patient 10) treated by coiling for a large unruptured top of the basilar artery (BA-top) aneurysm. A, Axial minimum intensity projection (MinIP) image of postcoiling FSE-MRC. The encircled arrow indicates the viewing position for the following 3D images. $B$, Axial MIP image of postcoiling TOF-MRA. C, Axial MIP image of postcoiling silent MRA. D, Postcoiling 3D FSE-MRC image. E, Postcoiling 3D TOF-MRA image. Note the gap (asterisk) of the parent artery and irregularity of the neck. F, Postcoiling 3D silent MRA image. Parent arteries and the coiled neck are clearly visualized. G, DSA image at the completion of coiling. Note that the configuration of the neck complex with remnants and parent arteries is depicted with the shadow of the coiled dome. $H$, A 3D multifusion image of postcoiling TOF-MRA and FSE-MRC. I, A 3D multifusion image of postcoiling silent MRA and FSE-MRC. Note that the coiled neck complex with remnants is clearly visualized together with the coiled dome and adjacent brain parenchyma. Pl indicates the first segment of posterior cerebral artery; Lt, left; Rt, right; AP, anteroposterior; An, aneurysm. was visualized with the dome, together with adjacent brain parenchyma (Figs 1-3).

\section{Size and Volume of the Neck Remnants and Coiled Domes}

The On-line Table also provides the size and volume of the neck remnants obtained by TOF-MRA and silent MRA and the coiled domes from FSE-MRC. With TOF-MRA, the sizes of the remnants were not measured accurately due to an incomplete depiction of the neck or parent arteries due to metal artifacts. In contrast, the size and volume of the remnants were obtained by silent MRA, and the size and volume of the domes were obtained by FSE-MRC. Although potential remodeling after coiling during the mean time interval between the MRA/MRC and the DSA $(8.5 \pm 4.4$ months) may have affected the findings, the neck and dome complex was not visualized identically but was similar to that provided by the DSA obtained at the time of coiling. Neck remnants were depicted in 10 of the 12 cases, and we calculated the percentage of the remnant volume (ie, the residual filling volume at the neck) measured by the silent MRA to the dome volume (the whole volume of the coiled dome) by the FSE-MRC in each case: $2.3 \%$ $36.7 \%$. The clinical results showed complete cure in 2 patients, follow-up with remnants in 8 patients, and retreatment in 2 patients. test revealed that the visualization score of parent arteries and neck status for the silent MRA $(3.75 \pm 0.5)$ was significantly higher than that for TOF-MRA $(1.5 \pm 1.25)(P<.01)$. The interobserver agreement tested by the intraclass correlation coefficient was 0.955 for TOF-MRA and 0.941 for silent MRA and was interpreted as excellent.

\section{D Visualization of the Aneurysmal Neck and Dome Complex}

With the use of TOF-MRA, the coiled neck and parent arteries were depicted but not precisely visualized, apparently due to metal artifacts. In contrast, the silent MRA clearly delineated the aneurysmal neck with/without remnants and afferent and efferent parent arteries without serious metal artifacts. Additionally, the shape of the dome treated and packed by coils was depicted by the FSE-MRC with perianeurysmal anatomic structures including the brain parenchyma and cranial nerves.

With the 3D multifusion images reconstructed and composed of 3D silent MRA and 3D FSE-MRC, the morphologic status of the neck and parent arteries of the coiled aneurysm

\section{DISCUSSION}

Endovascular treatment involving coil embolization has been performed widely for cerebral aneurysms, but coil compaction and the recanalization of a coiled aneurysm frequently occur after this treatment. ${ }^{1,5,6,8}$ The remnants of the dome may be at risk of growing, and they thus require long-term vascular follow-up. . $^{1,3,8}$ When retreatment is being considered, the clear visualization of the neck remnants and the dome configuration is necessary in the follow-up examination. $3,4,6,8,11$

In the present study, we used silent MRA for the follow-up in patients with coil treatment for cerebral aneurysms. Silent MRA may provide a clear depiction of flowing blood within the vessels (eg, the aneurysmal neck with/without remnants and parent arteries) with minimal artifacts caused by metal substances so that the aneurysmal complex can be delineated without serious susceptibility artifacts. ${ }^{2,7,9,10,12}$ With the currently available imaging software, the entire shape of an aneurysm (including the neck, parent arteries, and dome) treated by coiling can be reconstructed and depicted in a single $3 \mathrm{D}$ image by 


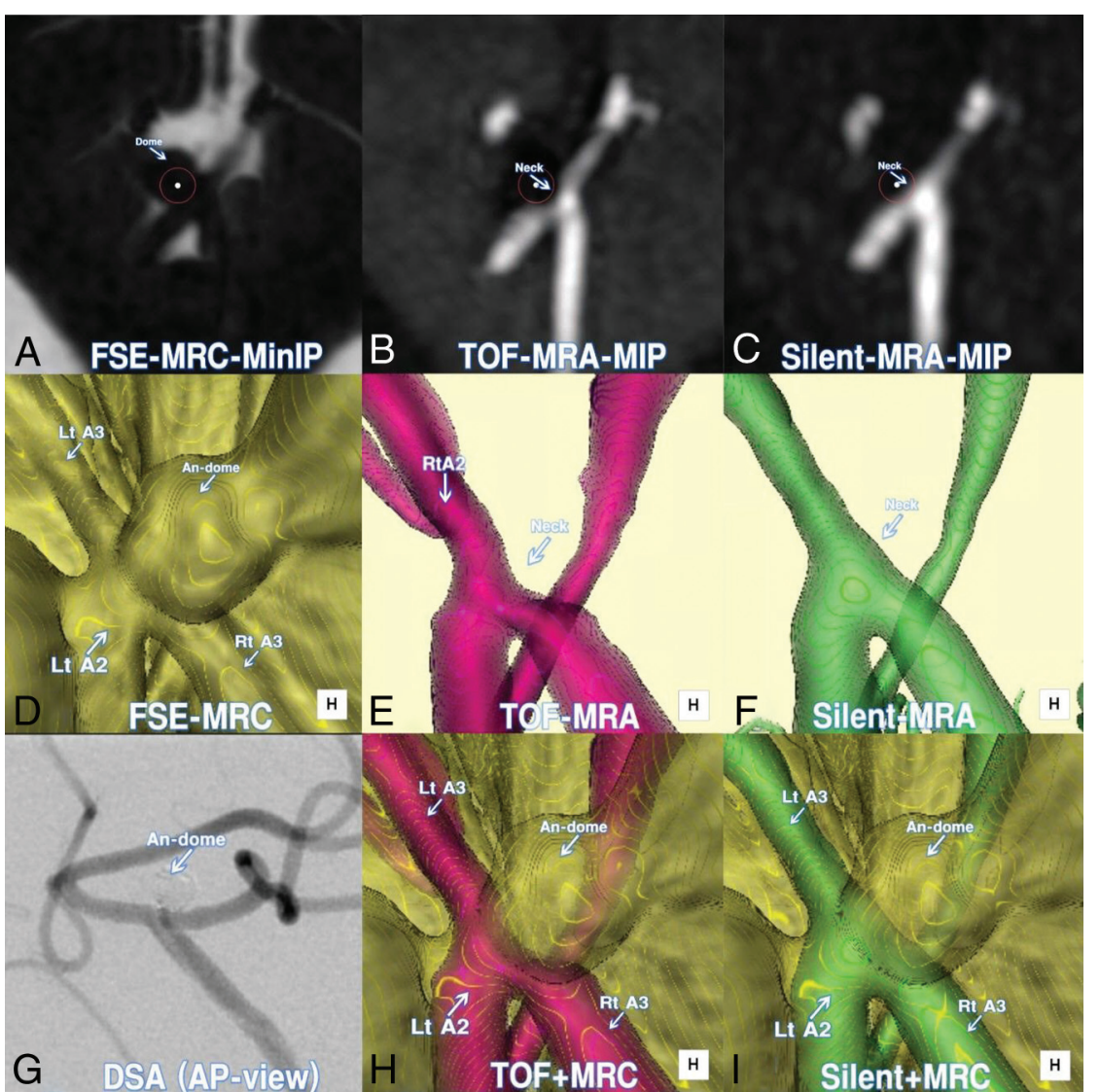

FIG 2. A 78-year-old woman (patient 6) treated by coiling for an unruptured distal anterior cerebral artery (A2-A3) aneurysm. A, Axial minimum intensity projection (MinIP) image of postcoiling FSE-MRC. B, Axial MIP image of postcoiling TOF-MRA. C, Axial MIP image of postcoiling silent MRA. D, Postcoiling 3D FSE-MRC image. E, Postcoiling 3D TOF-MRA image. Note the defects of the parent artery and the neck. F, Postcoiling 3D silent MRA image. Note that the parent arteries and coiled neck without remnants are clearly visualized. G, DSA image at the completion of coiling. Note that the configuration of the neck complex without remnants is depicted with the shadow of the coiled dome. $H, A$ 3D multifusion image of postcoiling TOF-MRA and FSE-MRC. I, A 3D multifusion image of postcoiling silent MRA and FSE-MRC. Note the complete occlusion of the neck without remnants together with the coiled dome. A2 indicates the second segment of the anterior cerebral artery; A3, the third segment of the anterior cerebral artery; An, aneurysm; $\mathrm{Lt}$, left; Rt, right; AP, anteroposterior.

the $3 \mathrm{D}$ multifusion imaging in combination with silent MRA and FSE-MRC described herein.

\section{Disadvantages of TOF-MRA}

TOF-MRA is a noninvasive technique used to obtain vessels-tosoft-tissue contrast by the flow of the spins within the blood vessels. The arterial geometry and patency obtained by TOF-MRA with/without contrast media do not represent the luminal morphology as depicted by DSA and CTA, but they do represent the flow voids caused by an inflow effect that are related mainly to peak systolic flow velocities within the vessel lumen during data acquisition. ${ }^{13-15}$ TOF-MRA may be affected by several factors, such as the loss of signal intensity that occurs from spin saturation effects due to slow flow and/or phase dispersion due to disturbed and complex flow. Magnetic susceptibility and radiofrequencyshielding artifacts from metal substances inevitably occur. ${ }^{10,16}$ As a result, it may be difficult to depict the precise configuration of the neck and parent artery of coiled aneurysms with TOF-MRA with/without contrast media.

\section{Merits of Silent MRA}

Silent MRA is also known as zeroTE MRA or arterial spin-labeling MRA, ${ }^{2,7,9,10,12}$ and the environmental noise during acquisition is $<3 \mathrm{~dB}$ (compared with $>100 \mathrm{~dB}$ with TOFMRA). Silent MRA is a non-contrastenhanced form of MRA, and its use may eliminate the acute and late possible adverse events of the administration of gadolinium-based contrast agents in the body and brain. ${ }^{17,18}$ In contrast to TOF-MRA, silent MRA depicts the configuration of flowing blood within the arteries so that the angioarchitecture of the aneurysmal neck complex is depicted without the contamination of veins, cranial base bones, and serious metal artifacts from coil materials. ${ }^{2,7,9,10}$ It is reported that silent MRA can visualize the flow in an intracranial stent more effectively than TOF-MRA. ${ }^{2}$ The details of the algorithm of silent MRA using the Silenz sequence were not disclosed by GE Healthcare; however, an ultrashort TE $(0.016 \mathrm{~ms})$ is used with arterial spinlabeling tagging and many spokes per segment for scanning. A preparation pulse is used at the carotid artery, and the data acquisition of the whole brain is based on a $3 \mathrm{D}$ radial scan. Before the labeling pulse, a control image without labeling is scanned first, followed by the labeled image. Subtraction of the control from the labeled image creates an angiogram (T1WI) that consists of pure flowing blood within the vessels.

In silent MRA, the ultrashort TE can minimize the phase dispersion of the labeled blood flow signal in the voxel space and decrease magnetic susceptibility artifacts. ${ }^{2,7,9,10}$ Consequently, silent MRA has much merit for depicting the arterial geometry at the neck as the flowing blood signal within the vessels with minimal metal artifacts. Additionally, due to the reduction of the blood flow saturation effect, silent MRA can depict slow flows, multidirectional flows, whirlpools, and turbulence flows more clearly compared with TOF-MRA.

Regarding the image processing, the maximum intensity projection images reconstructed from silent MRA usually show blurred margins of the vessels compared with TOF-MRA. The volumetric dataset must be selected using a distinct threshold that corresponds to the size of the vessels and borderline in the process of reconstructing $3 \mathrm{D}$ images. ${ }^{13-15}$ In the present study, a signal intensity threshold of $>300$ was used for the arteries. With this selected dataset, the outer wall configuration of the aneurysmal neck complex with coils can be depicted clearly without serious metal artifacts.

AJNR Am J Neuroradiol 40:802-07 May 2019 www.ajnr.org 

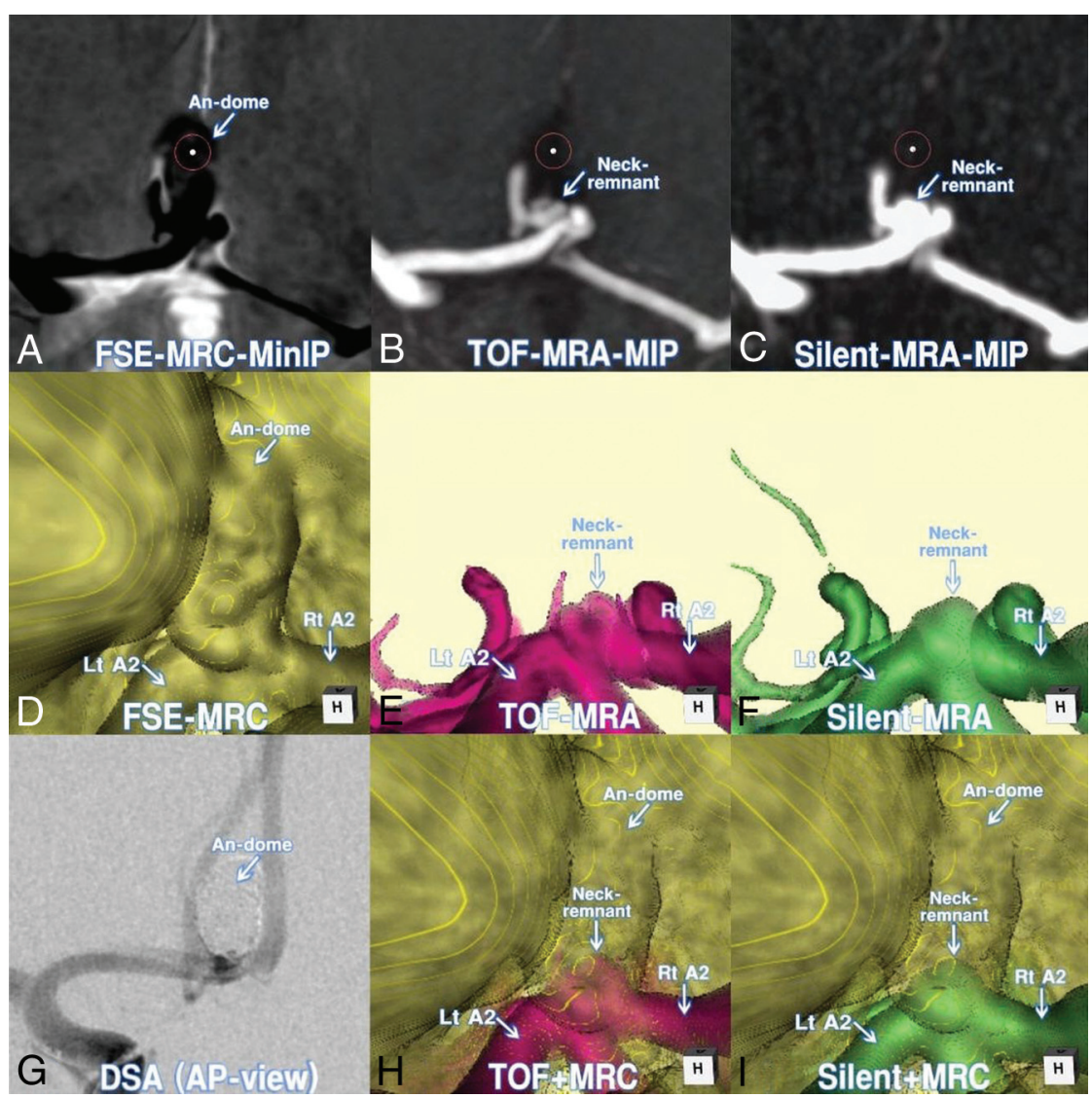

FIG 3. A 58-year-old man (patient 3 ) treated by endovascular coiling for an unruptured AComA aneurysm. A, Axial minimum intensity projection (MinIP) image of postcoiling FSE-MRC. $B$, Axial MIP image of postcoiling TOF-MRA. C, Axial MIP image of postcoiling silent MRA. D, Postcoiling 3D FSE-MRC image. E, Postcoiling 3D TOF-MRA image. Note the minor defects at the parent artery and the neck. F, Postcoiling 3D silent MRA image. Note that the parent arteries and coiled neck are clearly visualized. G, DSA image at the completion of coiling. Note the configuration of the neck complex with the shadow of the coiled dome. $H$, A 3D multifusion image of postcoiling TOF-MRA and FSE-MRC. I, A 3D multifusion image of postcoiling silent MRA and FSE-MRC. Note the clear visualization of the coiled neck complex with remnants, together with the coiled dome and adjacent brain parenchyma. Rt indicates right; Lt, left; AP, anteroposterior.

\section{Merits of FSE-MRC}

We did not use so-called T2 high-resolution images such as the FIESTA sequence for MRC. ${ }^{19}$ Because all the intracisternal anatomic structures are uniformly depicted as low signal intensity, it may be difficult to distinguish the boundary of an aneurysmal dome from the adjacent brain parenchyma and cranial nerves.

In the present study, we used FSE-MRC, a traditional heavily T2-weighted 3D FSE sequence, with a relatively short TR/TE (1900/100 ms). FSE-MRC obtained by a 3T unit with a wide bandwidth can depict the outer wall configuration of the intracisternal aneurysmal complex and perianeurysmal anatomic structures with thin sections and a high signal-to-noise ratio. ${ }^{13-15}$ An aneurysmal dome and parent arteries are depicted as a heavily dark area, and brain parenchyma and cranial nerves are depicted as a moderately dark area. These are well-contrasted to the surrounding CSF as a profoundly bright area. With FSE-MRC, the entire aspect of the aneurysmal dome and neck complex treated by coils is clearly visualized without metal artifacts. The contour of the coiled dome can thus be distinguished from the surrounding structures including adjacent brain parenchyma and cranial nerves.

\section{Merits of 3D Multifusion Imaging with Silent MRA and FSE-MRC}

Instead of using TOF-MRA and FIESTA-MRC, we reconstructed and combined silent MRA and FSE-MRC images by means of $3 \mathrm{D}$ multifusion imaging. ${ }^{13-15}$ Because the anatomic structure of a coiled aneurysm is complicated, it may be difficult to acquire a precise understanding of the architecture of the aneurysmal neck and parent arteries by simply reviewing $\geq 1$ of the source images displayed in 2D. Silent MRA may depict the coiled neck with/without remnants, and FSE-MRC delineates the dome and adjacent brain parenchyma after coiling treatment for cerebral aneurysms. The 3D reconstruction of silent MRA in combination with FSEMRC may provide the clear visualization of the aneurysmal complex with the coiled neck and dome in a single $3 \mathrm{D}$ image.

Our present results indicate that $3 \mathrm{D}$ multifusion images with silent MRA and FSE-MRC can provide the residual filling volume at the neck (remnant) with respect to the whole volume of the coiled dome (dome volume). This finding could be used in the decision regarding whether to conduct re-embolization. The size and volume of the remnant together with the coiled dome may also distinguish coil compaction from regrowth of the aneurysm. Consequently, visualization of the neck and dome with this imaging is useful for follow-up evaluations of patients treated with coiling for cerebral aneurysms.

\section{Limitations and Perspectives Regarding 3D Multifusion Imaging with Silent MRA and FSE-MRC}

Several limitations may exist in the application of 3D multifusion imaging with silent MRA and FSE-MRC. The sample size in the present study was small $(n=12)$, and the cases were limited to those of simple coil embolization. Cases with various types of stent- or flow-diverter-assisted coil embolization should be tested. In addition, silent MRA can depict the coiled aneurysmal neck complex with minimal metal artifacts via an ultrashort TE. However, a potential limitation is the occurrence of magnetic susceptibility and radiofrequency-shielding artifacts due to certain materials containing pure ferromagnetic substances such as a platinum alloy. ${ }^{7,11,16}$

Moreover, in the process of reconstructing 3D multifusion imaging, the size and volume of the neck and dome rendered for each 3D image may change according to the threshold value se- 
lected from an opacity chart of the datasets. ${ }^{13-15}$ It may be difficult to decide the optimal threshold values for the geometry of the aneurysmal neck for silent MRA and the dome for FSE-MRC in each individual subject. In this study, we determined the shape of arteries using the signal intensity threshold of $>300$ for silent MRA, $>400$ for TOF-MRA, and $<1200$ for FSE-MRC. By means of $3 \mathrm{D}$ multifusion imaging, an appropriate threshold for each TOF-MRA, silent MRA, and FSE-MRC can be determined by adjusting the shape of the objects, including parent arteries, neck, and dome of the coiled aneurysm by fusing 3 different images. In addition to the images of simple TOF-MRA, silent MRA, and FSE-MRC, 3D multifusion imaging may make a contribution by allowing the incorporation of all the views in a single $3 \mathrm{D}$ fused image.

Prospectively, we suggest that 3D multifusion imaging obtaining the pre- and posttreatment images with silent MRA and FSEMRC may be useful to confirm the initial success of coiling for cerebral aneurysms. Additionally, sequential follow-ups may depict changes in the shape of the coiled neck and dome, which may inform the timing of retreatment. More research is required to refine and validate the imaging techniques described herein.

\section{CONCLUSIONS}

Silent MRA can depict the configuration of the coiled neck and parent arteries without serious susceptibility artifacts. FSE-MRC can delineate the shape of the coiled dome together with adjacent brain parenchyma. In this small feasibility study, 3D multifusion imaging of silent MRA and FSE-MRC provided good visualization of key features of coiled aneurysms. This technique may be useful in the follow-up of coiled aneurysms.

\section{ACKNOWLEDGMENTS}

We thank Ms Megumi Sasaki, radiologic technician, and Ms Kana Murakami, laboratory technologist, Ryofukai Satoh Neurosurgical Hospital, for conducting the MR imaging examinations.

\section{REFERENCES}

1. Binning MJ, Veznedaroglu E. Aneurysm remnants after coiling and clipping: considerations in surgical and endovascular treatment options. Neurosurgery 2015;62:103-06 CrossRef Medline

2. Irie R, Suzuki M, Yamamoto M, et al. Assessing blood flow in an intracranial stent: a feasibility study of MR angiography using a silent scan after stent-assisted coil embolization for anterior circulation aneurysms. AJNR Am J Neuroradiol 2015;36:967-70 CrossRef Medline

3. Jabbarli R, Pierscianek D, Wrede K, et al. Aneurysm remnant after clipping: the risks and consequences. J Neurosurg 2016;125:1249-55 CrossRef Medline

4. Kaufmann TJ, Huston J 3rd, Cloft HJ, et al. A prospective trial of $3 \mathrm{~T}$ and 1.5T time-of-flight and contrast-enhanced MR angiography in the follow-up of coiled intracranial aneurysms. AJNR Am J Neuroradiol 2010;31:912-18 CrossRef Medline
5. Pierot L, Portefaix C, Gauvrit JY, et al. Follow-up of coiled intracranial aneurysms: comparison of 3D time-of-flight MR angiography at $3 \mathrm{~T}$ and $1.5 \mathrm{~T}$ in a large prospective series. AJNR Am J Neuroradiol 2012;33:2162-66 CrossRef Medline

6. Serafin Z, Strześniewski P, Lasek W, et al. Comparison of remnant size in embolized intracranial aneurysms measured at follow-up with DSA and MRA. Neuroradiology 2012;54:1381-88 CrossRef Medline

7. Shang S, Ye J, Luo X, et al. Follow-up assessment of coiled intracranial aneurysms using zTE MRA as compared with TOF MRA: a preliminary image quality study. Eur Radiol 2017;27:4271-80 CrossRef Medline

8. Shankar JJS, Lum C, Parikh N, et al. Long-term prospective follow-up of intracranial aneurysms treated with endovascular coiling using contrast-enhanced MR angiography. AJNR Am J Neuroradiol 2010;31:1211-15 CrossRef Medline

9. Takano N, Suzuki M, Irie R, et al. Usefulness of non-contrast-enhanced MR angiography using a silent scan for follow-up after Yconfiguration stent-assisted coil embolization for basilar tip aneurysms. AJNR Am J Neuroradiol 2017;38:577-81 CrossRef Medline

10. Takano N, Suzuki M, Irie R, et al. Non-contrast-enhanced silent scan MR angiography of intracranial anterior circulation aneurysms treated with a low-profile visualized intraluminal support device. AJNR Am J Neuroradiol 2017;38:1610-16 CrossRef Medline

11. Wallace RC, Karis JP, Partovi S, et al. Noninvasive imaging of treated cerebral aneurysms, Part I: MR angiographic follow-up of coiled aneurysms. AJNR Am J Neuroradiol 2007;28:1001-08 CrossRef Medline

12. Alibek S, Vogel M, Sun W, et al. Acoustic noise reduction in MRI using silent scan: an initial experience. Diagn Interv Radiol 2014;20: 360-63 CrossRef Medline

13. Satoh T, Onoda K, Tsuchimoto S. Visualization of intraaneurysmal flow patterns with transluminal flow images of 3D MR angiograms in conjunction with aneurysmal configurations. AJNR Am J Neuroradiol 2003;24:1436-45 Medline

14. Satoh T, Omi M, Osako C, et al. Visualization of aneurysmal contours and perianeurysmal environment with conventional and transparent 3D MR cisternography. AJNR Am J Neuroradiol 2005; 26:313-18 Medline

15. Satoh T, Omi M, Osako C, et al. Influence of perianeurysmal environment on the deformation and bleb formation of the unruptured cerebral aneurysm: assessment with fusion imaging of 3D MR cisternography and 3D MR angiography. AJNR Am J Neuroradiol 2005; 26:2010-18 Medline

16. McFadden JT. Magnetic resonance imaging and aneurysm clips: a review. J Neurosurg 2012;117:1-11 CrossRef Medline

17. Kanda $\mathrm{T}$, Ishii $\mathrm{K}$, Kawaguchi $\mathrm{H}$, et al. High signal intensity in the dentate nucleus and globus pallidus on unenhanced T1weighted MR images: relationship with increasing cumulative dose of a gadolinium-based contrast material. Radiology 2014; 270:834-41 CrossRef Medline

18. Quattrocchi CC, van der Molen AJ. Gadolinium retention in the body and brain: is it time for an international joint research effort? Radiology 2017;282:12-16 CrossRef Medline

19. Chávez GC, De Salles AAF, Solberg TD, et al. Three-dimensional fast imaging employing steady-state acquisition magnetic resonance imaging for stereotactic radiosurgery of trigeminal neuralgia. $\mathrm{Neu}$ rosurgery 2005;56:E628 CrossRef Medline 